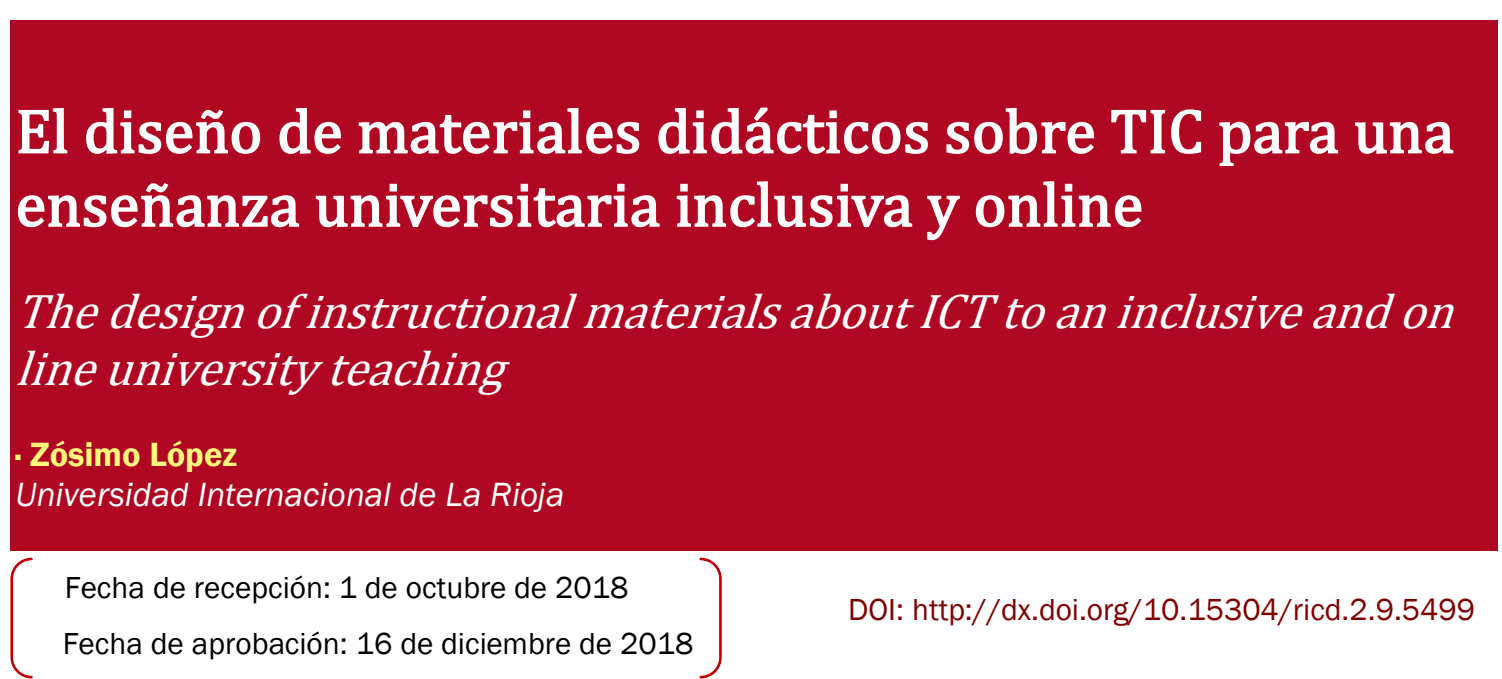

\title{
NOTAS BIOGRÁFICAS
}

Zósimo López es doctor en Comunicación e Industrias Creativas por la Universidad de Santiago de Compostela y profesor de Didáctica de la Lengua en la Universidad Internacional de La Rioja (UNIR).

Contacto: zosimo.lopez@unir.net

\section{Resumen}

El objetivo de este trabajo consiste en analizar el proceso de construcción de los materiales didácticos para una asignatura vinculada con la didáctica de tecnologías aplicadas impartida en un entorno en enseñanza online. En primer lugar se plantea un marco teórico centrado en los desafíos que genera la elaboración de materiales didácticos sobre tecnologías y el escollo que supone crearlos para su puesta en práctica en un entorno online de acuerdo con los paradigmas educativos que derivan de la enseñanza a distancia. Con respecto a la propuesta tomaremos como muestra el diseño de los materiales didácticos de la asignatura La Aplicación de las TIC y las TAC en la Enseñanza de la Lengua, parte del plan de estudios del Máster Universitario en Didáctica de la Lengua en Educación Infantil y Primaria que se imparte de forma virtual en la Universidad Internacional de La Rioja, orientada a ofrecer al estudiante los conocimientos necesarios para entender tanto las TIC como las TAC y, asimismo, su aplicación a la didáctica de la lengua en el aula con alumnos de Educación Infantil y Primaria de diferentes contextos educativos. Por último se ofrecen las conclusiones, en las que se constatará la idea de haber creado y puesto en práctica unos materiales de carácter inclusivo.

\section{Abstract}

The aim of this work consists in analyze the process of construction of the didactic materials for a subject linked with the didactics of technologies applied in an on-line teaching environment. At the first place, it plans a theoretical frame focused in the challenges that generates the preparation of didactic materials on technologies and the obstacle that supposes to create them for put into practice in some on-line environment according to the educational paradigms that generates the distance education. Regarding the proposal we will take like sample the design of the didactics materials from the subject La Aplicación de las TIC y las TAC en la Enseñanza de la Lengua. This subject is part of the plan of studies of the Master Universitario en Didáctica de la Lengua en Educación Infantil y Primaria which is taught in virtual mode at the Universidad Internacional de La Rioja. This subject is oriented to offer to the student the necessary knowledge to understand how to 
apply the ICT to the didactics of the language in the classroom with students of Early childhood and Primary Education in different educational contexts. Finally, we will offer the conclusions, in which we will confirm the idea of having created and put into practice some materials of inclusive character.

\section{Palabras clave}

Material didáctico, tecnologías, enseñanza online, didáctica de la lengua, inclusión.

\section{Keywords}

Educational materials, technologies, e-learning, language instruction, inclusion.

\section{Sumario}

1. Introducción

2. Marco teórico y estado de la cuestión

3. Método y materiales

4. Evaluación y resultados conseguidos

5. Conclusiones

\section{Contents}

1. Introduction

2. Theoretical frame and status of the issue

3. Methods and tools

5. Assessment and results

6. Conclusions 


\section{INTRODUCCIÓN}

Tanto la educación como la gestión del conocimiento han sufrido una revolución en lo que se refiere a métodos y a herramientas con la entrada en la era digital y el florecimiento y desarrollo de las tecnologías; esta situación ha generado nuevos formatos, conceptos y esquemas de pensamiento que deben saber aplicarse a las distintas asignaturas que integran los programas de estudio que conforman el sistema educativo español en todos sus niveles. Los diferentes currículums educativos nos revelan, efectivamente, la importancia de las TIC y, consecuentemente, en la enseñanza superior se hace necesario incorporar las tecnologías a los programas de estudio de grado y máster de las universidades españolas presenciales 0 a distancia. La creación de materiales didácticos para la didáctica de tecnologías puede presentar, sin embargo, algunos problemas derivados de la propia naturaleza caduca de los recursos y las herramientas tecnológicas, de su aplicación a disciplinas concretas pero también de los nuevos paradigmas didácticos que generan los entornos de enseñanza virtual.

En este trabajo nos proponemos analizar de qué manera puede el docente concebir una asignatura sobre la enseñanza de tecnologías aplicadas a la didáctica de la lengua para un entorno de enseñanza virtual y cómo esta se lleva a la práctica. A efectos del análisis tomaremos como muestra el diseño de los materiales didácticos de la asignatura La Aplicación de las TIC y las TAC en la Enseñanza de la Lengua, parte del plan de estudios del Máster Universitario en Didáctica de la Lengua en Educación Infantil y Primaria que se imparte de forma virtual en la Universidad Internacional de La Rioja (UNIR). Esta materia está orientada a ofrecer al estudiante los instrumentos y conocimientos necesarios para entender tanto las TIC como las TAC y, asimismo, para saber aplicarlas a la didáctica de la lengua en el aula con alumnos de Educación Infantil y Primaria de diferentes contextos educativos.

\section{MARCO TEÓRICO Y ESTADO DE LA CUESTIÓN}

Desde mediados de los años 80 uno de los temas más relevantes de la agenda política estatal y autonómica educativa en España ha sido la incorporación de las TIC (Area, 2012, p. 7); a ella deben sumarse, asimismo, las iniciativas que han llevado a cabo los propios centros educativos. En este sentido resulta relevante considerar la presencia de las tecnologías tanto en el Real Decreto 1630/2006, de 29 de diciembre, por el que se establecen las enseñanzas mínimas del segundo ciclo de Educación infantil como en el Real Decreto 126/2014, de 28 de febrero, por el que se establece el currículo básico de la Educación Primaria, los dos niveles educativos que nos interesan en el presente artículo.

En el primer texto curricular las tecnologías se mencionan en el bloque denominado Conocimiento del entorno y, dentro de este, su importancia se destaca como elemento que forma parte de la realidad de los niños y niñas y se aconseja que estos conozcan su papel y se inicien en su uso. Sin embargo conforman también un apartado de contenido en sí mismo dentro del bloque Lenguajes: Comunicación y representación denominado Lenguaje audiovisual y tecnologías de la información y la comunicación.

Por su parte, el currículum de Educación Primaria menciona la tecnología como parte de una competencia más amplia: Competencia matemática y competencias básicas en ciencia y tecnología. También cita su utilización para el aprendizaje en tanto que objetivo y como elemento transversal y, por tanto, se alude a ella en los contenidos y estándares de aprendizaje de todas las asignaturas que integran este nivel educativo en sus distintas etapas.

La integración de las TIC en el sistema educativo español implica, no obstante, algunas dificultades que atañen tanto a la formación del profesorado como a la falta de medios técnicos lo que genera una posible desigualdad. En un artículo reciente Colás, de Pablos, y Ballesta (2018, p. 2) se refieren a los problemas que suscita la integración de las TIC en el sistema educativo. A este respecto mencionan la insuficiente preparación del profesorado, tanto desde un punto vista instrumental como didáctico, para ponerlas en práctica en su quehacer diario. En este sentido son muchos los trabajos que han insistido en la importancia de la formación del profesorado para una correcta integración de las TIC (Castañeda, Esteve y Adell, 2018 , p. 20) o un desarrollo de la, llamada en los últimos años, competencia digital docente (Castañeda et al,. 2018, p. 2).

En otras ocasiones las dificultades no derivan de la formación que han recibido los docentes sino de la nula o pobre accesibilidad a Inter- 
net, de la ausencia de medios técnicos en un centro, o bien de su falta de mantenimiento por carecer el centro de personal destinado a tal fin (García-Valcárcel y Tejedor, 2010, p. 127), Io que genera distintos niveles de implantación de las TIC en el sistema educativo y, por tanto, una desigualdad.

Dados estos presupuestos, los programas formativos de grado y máster dedicados a la formación de profesorado de Educación Infantil y Primaria, como es el caso que aquí nos ocupa, deben contemplar la integración de asignaturas centradas en la enseñanza de TIC aplicadas a las distintas disciplinas objeto de estudio en el programa, con el objetivo de cumplir las exigencias del currículum, pero también para la correcta integración de las TIC en el aula.

La creación de los materiales didácticos para las asignaturas relacionadas con TIC entraña, sin embargo, algunos problemas. Su desafío principal se encuentra en la rápida obsolescencia de los materiales para utilizar en el aula; entre otros motivos, por la enseñanza de aplicaciones concretas que, en un breve plazo de tiempo, quedarán desfasadas.

Sin embargo, este proceso se complica cuando la modalidad de enseñanza no es presencial sino a distancia, debido a la naturaleza e implicaciones que ocasiona la segunda. El hecho de desconocer u olvidar sus particularidades supone que, en muchas ocasiones, la producción de los materiales didácticos se aborda de forma intuitiva (Calderone y González, 2016, p. 25) y, como consecuencia, una gran parte de ellos no responden a "los objetivos de enseñanza y aprendizaje, al contexto en el que se aplicará y/o a las particularidades técnicas y comunicativas de la tecnología seleccionada" (Cabero, 2010, p. 32).

A este respecto es importante recordar el extenso debate que se ha generado sobre el modelo de enseñanza que, desde un punto de vista técnico, exigen los entornos virtuales como del que requieren desde un punto de vista pedagógico. En este contexto, resulta de enorme relevancia la evaluación y reflexión sobre las propuestas de contenidos educativos, cómo estos se ajustan al espacio de enseñanza al que se dirigen y si contemplan el proceso de enseñanza-aprendizaje en toda su complejidad (Mauri, Onrubia, Coll y Colomina, 2016, p. 2). En definitiva, interesa considerar cómo la génesis del material didáctico para los entornos virtuales es, "un fenómeno poliédrico en el que se entremezclan distintas dimensiones que van mucho más allá del mero cambio de soporte tecnológico"
(Area, 2017, p. 17). Así pues, la transformación digital implica importantes cambios a nivel técnico, pedagógico, pero también en lo que se refiere a los papeles del docente y el discente y, asimismo, en lo que concierne a la creación y consumo de los materiales didácticos (Area, 2017, p. 24).

En este sentido puede recordarse, como indica Dorfsman (2018, pp. 2-3), el desafío que representa el hecho adecuar los contenidos de una materia a un espacio de enseñanza virtual, un contexto que, forzosamente, exige replantear la práctica docente por cuanto obliga a tener un conocimiento de los destinatarios de la asignatura.

Para el caso que nos atañe, y más tarde veremos, importa, por tanto, tener en cuenta la coincidencia en un mismo espacio de estudiantes de diferentes orígenes, culturas, edades, lo que en ocasiones podría suponer un hándicap en la alfabetización digital, y formaciones académicas. A ello debe sumarse que los futuros docentes que estamos formando van a ejercer su práctica en entornos muy diferentes con accesos desiguales a la tecnología, por lo tanto a la hora de planificar nuestros contenidos educativos debemos considerar un doble entorno: el que pertenece a nuestros alumnos de forma directa, como indica Dorfsman (2018), pero también los entornos hipotéticos en los que ellos podrían moverse en tanto que docentes.

A esta cuestión debe añadirse cómo el aprendizaje en entornos virtuales conlleva un proceso de construcción, puesto que lo que el alumno aprende en este espacio no es "simplemente una copia o reproducción de lo que en ese entorno se le presenta como contenido a aprender, sino una reelaboración de ese contenido mediada por la estructura cognitiva del aprendiz" (Onrubia, 2016, pp. 3-4 ); esto obliga a diceñar entornos y materiales de enseñanza y aprendizaje que se alejen del modelo transmisivo de enseñanza y que otorguen suficiente importancia a los procesos constructivos por parte del aprendiz de manera que este llegue a un aprendizaje significativo (Zapata, 2016, p. 13). Esta circunstancia la favorece, por ejemplo, cierto tipo las actividades; asimismo debe fomentarse la creación de contextos en los que el profesor pueda prestar su ayuda a los alumnos para que estos puedan llevar a cabo, con éxito, el proceso de aprendizaje constructivista (Rocha Fernandes y Ferreira, 2012, p. 99) como, por ejemplo, podemos hacer a través de los foros (Onrubia, 2016, p. 8). Así pues tanto el destinatario como el entorno de enseñanza-apren- 
dizaje y su modelo condicionan de forma determinante la naturaleza de los materiales didácticos que se crean para cada asignatura.

En primer lugar, estos deben estar ideados con una intencionalidad educativa en función del destinatario, puesto que el hecho de conocer al receptor permite al autor "determinar el estilo, la complejidad sintáctica o léxica del texto, la necesidad de utilizar recursos que contribuyan a la legibilidad del mismo, entre otros elementos" (Rey, Barbosa y Gómez, 2003, p. 58).

En segundo lugar, los materiales deben considerar la estrategia pedagógica que se desea seguir. En este sentido resulta evidente que estos han de promover y estimular el aprendizaje del alumnado. Por este motivo, al referirse a los criterios que deben considerarse a la hora de secuenciar los contenidos de una materia Zapata $(2016$, p. 15) indica la importancia de considerar no solo el contenido sino también el modo en el que los alumnos construyen su conocimiento. Otro aspecto que debemos considerar en cuanto a la selección de contenidos para nuestros materiales será el de la inclusión, de manera que los más simples y generales se sitúen en primer lugar y los más concretos en segundo (Zapata, 2016. pp. 15-16).

En este contexto las actividades jugarán, asimismo, un papel determinante por cuanto estas ayudan a "entender, desarrollar y practicar habilidades y a aprender" (Rey et. al. 2003, p. 58). A este respecto, algunos autores han señalado cómo, a lo largo de la evolución del e-learning, además de a los contenidos, se ha dotado de gran significado a la realización de tareas o eactividades por parte del alumnado para el logro de los objetivos planteados en la asignatura y la construcción de su propio aprendizaje (Cabero, 2015, p. 9). Este método combina, por tanto, el contenido y su aplicación a las actividades (Silva, 2017). No obstante, es importante que las actividades que les propongamos a los alumnos sean lo suficientemente significativas en el proceso de formación que perseguimos. Muy interesantes son, en este sentido, los principios clave que debe regir el diseño de estas actividades y señala Cabero:

Que los alumnos perciban que las actividades se encuentren claramente relacionadas con los objetivos, competencias y capacidades que perseguimos que alcancen. - Que se les otorgue suficiente tiempo para su realización. - Que los criterios que vamos a utilizar para su evaluación los conozcan, y sean claros y entendibles. - Que no sean ni fáciles de realizar, ni muy complejas de resolver. - Que se encuentren contextualizadas. Que las instrucciones para su cumplimentación sean claras y entendibles. - Que los materiales que tengan que utilizar para su realización sean fácilmente localizables, y se encuentren a su disposición. Y que se ofrezcan actividades de diferente tipología. (Cabero, 2015, p. 13)

Dichas actividades, diseñadas para motivar y favorecer la comprensión, también proporcionan a los alumnos un indicativo de sus progresos y, por tanto, forman parte de la llamada evaluación formativa. Esta suele convivir, en los entornos de enseñanza a distancia, con la sumativa, que permite registrar los logros de los alumnos, suele realizarse al final y comporta una calificación. La combinación de ambas formas permite llevar a cabo una evaluación continua caracterizada por ser manejable, alentadora y motivante (Dorrego, 2016, p. 3).

En tercer lugar, los materiales que creemos deben evaluarse con la finalidad de averiguar si se ha logrado el efecto educativo para el cual se han concebido (Rey et al., 2003, p. 56).

Parece evidente, por lo tanto, que la preparación de una asignatura en línea exige una planificación al detalle que considere el contexto de impartición con todas sus implicaciones, el modelo técnico y pedagógico de la enseñanza distancia y, por supuesto, el diseño de los contenidos, las actividades, la evaluación a través de las actividades y la prueba final y la evaluación de los propios materiales.

\section{MÉTODO Y MATERIALES}

La Universidad Internacional de La Rioja (UNIR), institución en la que inscribe nuestra propuesta, cuenta en la actualidad con más de 10.000 alumnos internacionales. La universidad ofrece un modelo de enseñanza virtual que incluye clases online en directo que pueden, posteriormente, visualizarse en diferido; clases y foros para resolver dudas; clases magistrales y una serie de recursos de aprendizaje que permite caminar desde lo esencial (Ideas Clave) a lo complementario (material extra) y de lo teórico (lecturas) a lo práctico (actividades y test). Se favorece de este modo la flexibilidad en el recorrido por la gran cantidad de información de la que disponen, la utilización de material con distintos fines y objetivos o la implicación del lector en la toma de decisiones sobre lo que quiere aprender, todas ellas características de los materiales electrónicos interactivos (Sánchez, 2012, p. 5). A esto debe sumarse que se propone un sistema de evaluación continua inte- 
grada por las actividades formativas y el examen final.

El objetivo principal de nuestra propuesta didáctica fue la creación de un material docente para la asignatura La Aplicación de las TIC y las TAC en la Enseñanza de la Lengua que se imparte en el Máster Universitario en Didáctica de la Lengua en Educación Infantil y Primaria que se enseña en la UNIR.

Los destinatarios de este material didáctico fueron los 45 alumnos y alumnas que cursaron esta asignatura desde el 27 de marzo de 2017 al 16 de julio del mismo año. 21 de los cuales asistían a las clases virtuales desde Latinoamérica y 24 de ellos asistían a las clases virtuales desde España, lugares de los que procedían respectivamente. Estos alumnos tenían una media de edad de 36 años: los alumnos españoles tenían una media de 32 y los alumnos hispanoamericanos, una media de 39 años. El 85\% eran mujeres frente a un $15 \%$ que eran hombres.

Como antes indicábamos, el modelo de enseñanza a distancia en el que trabajamos obliga al docente al conocimiento de la diversa realidad social y educativa de los alumnos que integran el grupo, por cuanto esto favorece el proceso de enseñanza-aprendizaje. En el presente caso esta heterogeneidad se observa a través de los datos ofrecidos en el párrafo anterior, una diversidad que también afecta a los niveles de alfabetización digital del alumnado. Esta circunstancia implicaba la creación e impartición de una serie de contenidos abiertos y versátiles que permitiesen la incorporación de todo el grupo que cursase asignatura favoreciendo de este modo una educación inclusiva (UNESCO, 2008, p. 7), que podemos potenciar de forma clara con la enseñanza a distancia.

En relación con esta misma idea nos parecía importante también enseñar a nuestros discentes a ser docentes en contextos con diferentes grados de acceso a elementos tecnológicos; lo que implica desde un uso limitado de hardware hasta un acceso limitado o nulo a internet que conlleva un acceso limitado al conocimiento y, por lo tanto, un posible motivo de exclusión en un mundo interconectado. En este sentido ha de considerarse, por ejemplo, cómo las administraciones públicas que velan por ofrecer una equipación tecnológica no reparten de manera equitativa los elementos informáticos necesarios debido a múltiples y diferentes razones o de qué manera las casas editoriales (Aguerrondo, 2006, p. 2), responsables también en mayor o en menor medida de la creación de contenidos edu- cativos digitales, provisionan de hardware a algunos centros educativos pero no a otros.

Teniendo en cuenta esta situación, en los materiales de la asignatura La Aplicación de las TIC y las TAC en la Enseñanza de la Lengua se persigue explicar cuál es la esencia de la aplicación de estas tecnologías a la didáctica de la lengua en el aula de Infantil y Primaria. A este respecto, se ha tomado la decisión de no centrarse en conocimientos técnicos en programas y aplicaciones, de rápida caducidad, sino que el objetivo de la configuración del material didáctico de esta asignatura ha consistido en dotar a los alumnos de una visión estratégica sobre las posibilidades de estas tecnologías aplicadas a la educación y, más concretamente, a la didáctica de la lengua en las etapas de Infantil y Primaria. Por lo tanto hemos tratado de presentar, en las ideas clave, los conceptos más generales e inclusivos, dejando para el material extra, las lecturas y las actividades los aspectos más concretos.

Nuestro interés en este artículo científico se centra, por tanto, en desentrañar los procesos de creación de los recursos de aprendizaje ideados para la citada asignatura, además del examen de los procesos de evaluación a los que se somete tanto el alumnado como los mismos materiales y sus resultados.

Para el diseño de la propuesta, integrada por siete temas que incluyen ideas clave, una clase magistral por cada tema, el material extra y las actividades, se han tenido en cuenta las competencias que corresponden a esta asignatura contempladas en la memoria oficial del programa de máster aprobada por la ANECA, divididas en básicas, generales y específicas.

Como metodología para la construcción de estos materiales didácticos y siguiendo el objetivo de cumplir con las competencias anteriormente mencionadas en este trabajo, se han fijado las ideas clave correspondientes a los siete temas con sus lecciones magistrales y materiales complementarios. A tal efecto, tras consultar y analizar 164 fuentes documentales de carácter científico, se elaboraron unos materiales de 227 páginas que pueden describirse como sigue.

El primero de ellos (La enseñanza mediante TIC y TAC en Educación Infantil y Primaria) sirve como introducción a la asignatura y en él se aporta una visión general sobre las definiciones de TIC y TAC, Ios cambios que conlleva su aplicación en el aula (Expósito y Manzano, 2013; Coll, 2004; García y Ruíz de Adana, 2013; Minelli de 
Oliveira, s.f. y UNESCO, 2008), en qué consiste la Competencia Digital y cuál debería ser la actitud de los docentes frente al uso de las TIC y las TAC en el aula (Cabezas, Casillas y Pinto, 2014; Cortés, 2013; Enríquez, 2012).

El segundo (Introducción a las TIC y TAC en la enseñanza de Lengua) tiene como objetivo principal aportar al alumno una visión general de las TIC y TAC (Siraj-Blatchford, 2005) aplicadas a la didáctica de la Lengua Castellana; el tratamiento que se le da en distintos currículum educativos al uso de estas tecnologías (Martín y Marchesi, 2006), así como una breve explicación sobre los elementos básicos que conforman las tecnologías informáticas como son el hardware con orientación educativa (Asorey y Gil, 2009; Martín, 2013) y software con orientación educativa (Gewerc, 2002).

El tercero (El desarrollo de la competencia lingüística y la competencia digital del alumno de Infantil y Primaria) plantea la explicación de la competencia comunicativa y la competencia lingüística y cómo estas se deben implementar en el aula de Infantil y Primaria haciendo uso de las TIC y las TAC. Para finalizar, se introduce el concepto de la alfabetización multimodal (Kress, Jewitt, Ogborn y Tsatsarelis, 2001) y su repercusión a la hora de enseñar comunicación en las etapas educativas que nos ocupan.

En el cuarto (La aplicación de las TIC y las TAC en la enseñanza de Lengua en Infantil y Primaria) se manejan posibles contextos en los que se puede encontrar un docente de Lengua en Infantil y Primaria frente a las TIC y las TAC. Estos tienen relación con las características de los centros, con la importancia de la familia (Sáinz de Vicuña, 2009) en la alfabetización multimodal y digital del alumnado o las diferentes realidades que pueden afectar a la educación del niño, con la finalidad de identificar posibles aplicaciones de las TIC y las TAC en la enseñanza de Lengua para estas etapas (Farías y Araya, 2014; Ballestas, 2015). Asimismo, en este tema se introduce la problemática que ofrece la distinción entre las TIC y las TAC frente a la comunicación comercial (Urbina, 2006) en los medios de comunicación (Martí y Muñoz, 2008). Para finalizar este tema, se expondrá el papel que desempeña la Web 2.0 y la Web 3.0 como apoyo a la enseñanza de la lengua (Castaño, 2013).

El tema cinco (Los entornos virtuales de aprendizaje y la enseñanza de la Lengua en el aula de Infantil y Primaria) se centra en la educación semipresencial y la no presencial por medio de herramientas informáticas (EVA, PLE, MOOCs...) como uno de los elementos más im- portantes de las TAC (Boneu, 2007; Salmerón, Rodríguez, y Gutiérrez, 2010). En este sentido, se describen y analizan sus características, herramientas y usos, haciendo especial hincapié en el aprendizaje colaborativo en línea (Basilotta y Herrada, 2013), lo que constituye uno de los parámetros que favorece la educación virtual.

El objetivo principal del tema 6 (Fundamentos y recursos para el diseño de materiales audiovisuales para la enseñanza de la Lengua en Infantil y Primaria) es el de reflexionar acerca del papel que juega el docente en tanto que creador de contenidos (Belloch, S.F.; Cebrián, 2013) para enseñar Lengua a alumnos de las etapas de Infantil y Primaria. Por fin, en el último tema (La evaluación de la competencia lingüística a través de las TIC y TAC en Infantil y Primaria) se ofrecen algunas ideas sobre cómo aplicar las tecnologías a la evaluación de Lengua en el aula de estas etapas (Fernández y Cebreiro, 2003).

Como antes hemos indicado, además de los contenidos señalados, cada uno de los temas se acompaña, en primer lugar, de una lección magistral de quince minutos de duración que permiten al alumno profundizar en algún aspecto vinculado con el tema explicado. Por ejemplo, la correspondiente al primer tema está centrada en la competencia digital fuera de la escuela y permite acceder a los alumnos a una realidad ajena a la escuela en la que dicha competencia resulta de vital importancia. En segúndo lugar, al final de cada tema se recomienda al alumno de lectura de dos artículos concretos sobre distintas ideas manejadas a lo largo del tema. Asimismo, y en tercer lugar, se ofrece al alumno la posibilidad de profundizar en determinados aspectos que se han tratado de manera más general a lo largo del tema mediante el acceso a publicaciones de carácter didáctico relacionadas con la aplicación de las tecnologías en el aula en espacios educativos concretos de distintas partes del mundo. En cuarto lugar se aporta al alumno entre dos y tres recursos web vinculados de forma general o específica con el tema correspondiente. Por último, se guía al alumno facilitándole un glosario de los conceptos más relevantes tratados en el tema.

\section{EVALUACIÓN Y CONTENIDOS CONSEGUIDOS}

Como instrumentos de la evaluación continua la UNIR establece 2 asistencias obligatorias a las clases virtuales en modo sincrónico, 2 asistencias obligatorias a las clases virtuales en 
modo asincrónico, 7 pruebas tipo test (una por tema), 5 trabajos, la participación en un foro obligatorio que se plantea como actividad y una prueba final con forma de examen.

Los primeros elementos de recopilación de información sobre el avance en la adquisición de conceptos por parte de los alumnos han sido los cinco trabajos y el foro obligatorio. La mayor parte del alumnado de la asignatura que nos interesa está constituido por profesionales de la enseñanza en activo y el fragmento restante está integrado por estudiantes que aspiran a entrar en este mercado laboral. En estos trabajos académicos se le proponen al estudiante unos parámetros para realizar una investigación personal sobre diferentes temas, que relacionan las tecnologías con la didáctica de la lengua en educación Infantil y Primaria en espacios de aplicación reales, como son, por ejemplo, el conocimiento de la legislación vigente en materia de educación en relación con las TIC, la aplicación de las TIC a los contextos inmediatos, la innovación tecnológica o la evaluación por medio de tecnologías. Los 46 alumnos realizaron un total de 184 actividades: 151 trabajos y 33 asistencias al debate propuesto en el foro de la plataforma de la asignatura. Estas aportan una información suficiente para conocer los contextos, retos, debilidades y fortalezas a las que se enfrenta este alumnado a la hora de trabajar con tecnologías dentro de su presente o posible centro educativo tal y como se reflexiona a continuación.

Es posible, además, que uno de los pilares más importantes sobre el que se basa la fijación de contenidos de esta materia sean los cinco trabajos propuestos al alumnado y el foro. Todos ellos entrañan tareas auténticas y están propuestos de una manera constructivista, de forma que el primer trabajo sirviera para comprender el siguiente y que los sucesivos necesitaran conocimientos tratados en los anteriores. Se plantearon de forma que se trataran los siguientes conceptos: currículum y TIC, innovación, acceso a TIC, diseño de proyectos multimedia y evaluación y futuro de las TIC y las TAC. A continuación, y por orden de realización, se describen brevemente:

La primera actividad (Currículo, TIC y TAC: ¿cómo combinar esta tríada?) consistió en la realización de un trabajo sobre el tratamiento que reciben las TIC y las TAC en el ámbito de la Educación Infantil y Primaria en la región en donde trabaja o aspira a trabajar el estudiante. Este trabajo lo han realizado 39 alumnos y cabe señalar que en la puesta en común de los resultados en clase, a los alumnos españoles e incluso a los propios colombianos les llamó la atención que en este país no existieran unos lineamientos concretos para esta área, en este nivel de enseñanza. Otro punto de reflexión muy interesante fue el de comprobar en los documentos de estos alumnos la afirmación de que desde la documentación de la administración educativa se anima a la equipación y uso de las TIC pero, en la realidad, los docentes en activo afirmaban que no todos los centros escolares estaban equipados tecnológicamente de manera adecuada e incluso una alumna llegó a argumentar que "si un centro escolar no tiene luz, malamente podrán usar las TIC".

La segunda actividad (Impensables pensables posibilidades de las TIC y TAC) consistió en proponer nuevas posibilidades de aplicación de las TIC y las TAC en el campo de la didáctica de la Lengua. Por medio de esta actividad se pretendía evaluar la comprensión del concepto de estas tecnologías aplicadas a la educación. $\mathrm{Ha}$ sido realizado por 36 estudiantes y, según sus comentarios en clase, ha sido la más difícil debido a su abstracción. 15 de estos 36, esto es un $41 \%$ de los alumnos que realizaron esta actividad, no cumplió con el requisito principal que era el de explorar y analizar si las posibilidades que ofrece el hardware son las mejores en el ámbito de la didáctica de la lengua en las etapas de Infantil y Primaria. Esto puede poner de relieve que no muchos de estos estudiantes están acostumbrados a dinámicas de innovación o que, quizás, no están dotados de unos conocimientos mínimos relacionados con creatividad. Sin embargo, 6 alumnas consiguieron obtener la calificación máxima en esta prueba proponiendo innovaciones interesantes con aplicación al hardware con fines educativos en la materia y etapas que nos conciernen. Así con todo, se puede notar cierta diferencia entre los dos grupos de la clase a la hora de realizar esta tarea en donde el grupo 1 obtuvo una nota media de un 7.4 mientras que el grupo 2 tuvo un 5.81 , lo que lleva a pensar que quizás el segúndo grupo no está tan habituado a este tipo de actividades en donde la creatividad juega un papel importante.

La tercera actividad (TIC y TAC vs. la realidad en la enseñanza Infantil y Primaria) consistió en la realización de un análisis sobre el acceso que tienen los alumnos de Infantil y Primaria a las TIC y TAC en las diferentes realidades en donde los discentes de este máster tienen la posibilidad de trabajar como docentes de Lengua y Literatura. Cada alumno pudo configurar dicho análisis a partir de la realización de una encues- 
ta por parte del discente (ya diseñada por el docente) a una persona responsable de un centro escolar y a un docente del área de lengua. En esta encuesta se les preguntaba por ítems relacionados con la calidad del acceso a internet, la equipación TIC de la que disponía el centro escolar o las aulas en donde se impartía lengua, entre otros. Este trabajo estaba pensado para motivar a los alumnos a tener una razón para investigar e indagar sobre las equipaciones tecnológicas de los centros escolares de su entorno y así tener una visión práctica y real sobre cuál es el estado de la cuestión. Este trabajo lo realizaron 34 alumnos.

La cuarta (¿Qué forma tiene esta idea?) consistió en idear un recurso didáctico con formato audiovisual interactivo orientado a la didáctica de la Lengua en Educación Infantil y Primaria. En esta actividad se valoró especialmente la posible viabilidad del proyecto. Para realizarla los estudiantes tuvieron que idear, planificar y ejecutar un recurso orientado a explicar algún contenido concreto relacionado con la Lengua y dirigido a un curso concreto de Infantil o de Primaria, haciendo más hincapié en la argumentación en la que se fundamenta esta actividad que en su ejecución técnica. Este trabajo dotaba de gran libertad a los estudiantes para elaborar ideas a partir de los contenidos impartidos en este tema (relacionados con comunicación audiovisual y multimedia) y, aunque se mostraron diferentes aplicaciones informáticas en clase, en ningún momento se tomó como criterio de evaluación de este trabajo el manejo de una o más herramientas informáticas sino en lo que se puso más énfasis fue en la capacidad creativa a la hora de idear recursos didácticos. Llama la atención que solamente 22 alumnos (6 del grupo 1 y 16 del grupo 2) de un total de 45 han desarrollado esta tarea.

La quinta actividad (¿Qué tal lo he hecho, profe?) Esta última actividad consistió en idear un recurso digital orientado a la evaluación de la competencia lingüística de alumnos de Infantil y Primaria. Dicho recurso digital tenía que ser ideado teniendo en cuenta el currículum vigente y debía estar enfocado en alguna destreza comunicativa. Este trabajo, que solamente fue realizado por 11 alumnos, tenía como objetivo principal que el alumno fuera capaz de idear nuevos recursos, estrategias y/o metodologías para la evaluación por medio de las TIC y TAC.

Por su parte, el foro obligatorio, que lleva por título Primero las TIC, después las TAC... ¿Y después?, trata de incentivar a los alumnos a reflexionar sobre las posibilidades que pueden llegar a ofrecer las tecnologías estudiadas a lo largo del temario en el campo de la didáctica de la lengua en la educación Infantil y Primaria.

Además de la evaluación formativa, que podemos llevar a cabo a través de las actividades, la UNIR contempla también una evaluación sumativa a través del examen. Este se ha configurado de la siguiente manera: la primera parte está formada por siete cuestiones de comprensión de carácter abierto. Cada una de ellas está integrada por dos preguntas: a través de la primera ele alumno demostrará que ha asimilado algún concepto propuesto en el temario de la asignatura y, mediante la segunda, aportará un ejemplo de la aplicación práctica de ese concepto a la realidad laboral del docente. Sirva como ejemplo esta cuestión: ¿Qué es el hardware? ¿Qué tipo de hardware cree que es necesario para impartir lengua en una etapa de Infantil y Primaria? ¿Justifique su respuesta? En la segunda parte del examen se le plantea al alumnado un caso práctico a desarrollar. Para contestar satisfactoriamente a este caso práctico el discente deberá seguir una serie de pasos para diseñar una actividad con tecnologías aplicadas a la didáctica de la lengua con un alumnado de edades comprendidas en un rango determinado por el autor del examen. Realizar con éxito este diseño de actividad implica saber utilizar todos los conocimientos adquiridos a lo largo de esta asignatura, ya que es necesario mostrar un conocimiento del currículum, dominar las diferentes competencias, ser conscientes de los elementos informáticos que están al alcance del profesorado y del alumnado, tener una capacidad mínima de reflexión sobre por qué y cómo se utilizarán máquinas y aplicaciones y adecuar un sistema de evaluación a lo que se quiera diseñar y a la actividad docente que se quiera llevar a cabo.

Finalizado el proceso de evaluación de la asignatura podemos exponer que, en términos de calificación, los 46 alumnos han conseguido una nota media de 7,33 sobre 10 . No han superado la asignatura en convocatoria ordinaria 2 alumnos y 1 alumno no se presentó.

En tercer lugar, debemos tener en cuenta la evaluación realizada por parte del alumnado, lo que nos ayuda a valorar el grado de satisfacción con los materiales propuestos. Para ellos nos valemos del cuestionario configurado por la UNIR para conocer el grado de satisfacción de los alumnos sobre la docencia recibida que consta de 20 preguntas para puntuar sobre 10. Contamos con 15 cuestionarios contestados de los 46 enviados. De las 20 preguntas que conforman el 
cuestionario creemos que puede ser pertinente para este artículo traer a colación las tres que a continuación se revisan. El alumnado consultado ha puntuado la pregunta "El docente vincula los contenidos con la realidad" con un 9,5 sobre 10 . Estimamos que esta cuestión tiene su importancia debido a que los estudiantes aprecian una vertiente práctica en los contenidos propuestos en esta materia. Asimismo, la pregunta "He mejorado mi nivel en las competencias previstas para la asignatura" ha sido puntuada por parte del alumnado con un 9,3 sobre 10. Para finalizar, la pregunta "He mejorado mis conocimientos y habilidades en esta materia" ha sido puntuada con un 9,1 sobre 10. Estas dos últimas preguntas ayudan a constatar la efectividad de los materiales en cuanto a su planteamiento acertado lo que contribuye al desarrollo de la competencia digital de los futuros docentes.

\section{CONCLUSIONES}

La incorporación de las tecnologías a los programas de estudio de los diferentes niveles educativos del sistema español ha sido, en los últimos años, una prioridad de las administraciones y de los propios centros educativos. Estas forman parte, como hemos visto, de los currículums de Infantil y Primaria y, por lo tanto, deben protagonizar asignaturas individuales en los programas de grado y máster. Sin embargo la creación de materiales didácticos vinculados con asignaturas centradas en la enseñanza de tecnologías implica numerosos escollos; por ejemplo el vinculado con la rápida caducidad de las herramientas tecnológicas concretas. A los condicionantes inherentes a la naturaleza de las tecnologías deben sumarse, no obstante, aquellos que debemos considerar si la didáctica se imparte en un entorno de enseñanza superior a distancia, pues este supone un nuevo modelo no solo técnico sino también pedagógico que, obviamente, influye de forma determinante en la manera de crear los materiales didácticos de una asignatura $y$, por tanto, en el modo de impartirla.

Por estos motivos para la creación de materiales didácticos correspondientes a la asignatura La Aplicación de las TIC y las TAC en la Enseñanza de la Lengua se consideró el heterogéneo destinatario al que se dirigían los materiales, particularmente en lo que se refiere a su competencia digital. Teniendo en cuenta esta circunstancia se tomó la decisión de crear unos materiales de carácter inclusivo, en los que se ofreció al discente, a través de las Ideas Clave que integran los siete temas, los instrumentos y conocimientos necesarios para entender tanto las TIC como las TAC y, asimismo, aplicarlas en el aula con alumnos de Educación Infantil y Primaria de diferentes contextos educativos, bien en el entorno urbano y tecnológico bien en un contexto rural y aún con dificultades para acceder a tecnologías aplicadas a la educación. Lo que se ha perseguido en estos materiales es cumplir, por tanto, con la idea general propuesta por la UNESCO en relación con la forma equitativa de incluir las TIC en las aulas gracias a las cuales se lograrán cambios pedagógicos que mejoren el sistema educativo (Aguerrondo, 2006, p. 11). Adicionalmente, gracias a los materiales complementarios, integrados por las lecturas o los recursos web, se permitió al alumno profundizar o concretar en aplicaciones determinadas. De esta forma creemos haber ofrecido unos materiales de carácter flexible y, asimismo, personalizables. En este proceso de enseñanza-aprendizaje jugaron un papel fundamental las actividades, todas ellas de distintos tipo, planteadas desde un punto de vista constructivista, con intenciones motivadoras y vinculadas con la realidad de cada alumno. A través de ellas se pudo llevar a cabo una evaluación formativa. A ella debe unirse la sumativa que pudimos realizar gracias al examen final en el que cual tuvo una importancia significativa la aplicación práctica y didáctica de los conocimientos adquiridos. Parece importante destacar los resultados favorables de estas dos evaluaciones así como de la realizada por los alumnos con respecto a la calidad de asignatura y su desarrollo a través del cuestionario creado por la propia universidad, pues ambas nos ayudan a constatar la idoneidad de los materiales, aunque, por supuesto, resulta obvio estos están sujetos a revisión y actualizaciones permanentes que permitan mejorar la calidad educativa de esta asignatura en el contexto del Máster Universitario en Didáctica de la Lengua en Educación Infantil y Primaria.

\section{REFERENCIAS BIBLIOGRÁFICAS}

- Aguerrondo, I. (coord.) (2006). La integración de las Tecnologías de la Información y la Comunicación en los Sistemas Educativos. Buenos Aires: Sede UNESCO de Buenos Aires. Recuperado de: 
https://unesdoc.unesco.org/ark:/48223/pf000015 0785 .

- Area, M. (2012). Políticas educativas TIC en los sistemas escolares en Iberoamérica. Miradas desde las dos orillas. Campus Virtuales. 1 (1), 7-9. Recuperado de:

http://www.uajournals.com/campusvirtuales/jour nal/1/0.pdf.

- Area, M. (17 de diciembre de 2017). La metamorfosis digital del material didáctico tras el paréntesis Gutenberg. RELATEC: Revista Latinoamericana de Tecnología Educativa. 16 (2), 13-28. DOI: 10.17398/1695-288X.16.2.13.

- Asorey, E y Gil, J. (12 de noviembre de 2009). El placer de usar las TIC en el aula de infantil. $C E E$ Participación Educativa 12, 110-119. Recuperado de: https://goo.gl/L5pW7F.

- Ballestas, R. (2015). Relación entre TIC y la adquisición de habilidades de lectoescritura en alumnos de primer grado de básica primaria. Investigación \& Desarrollo. 23 (2), 338-368. Recuperado de DOI: 10.14482/indes.23.2.7398.

- Basilotta, V. y Herrada, G. (2013). Aprendizaje a través de proyectos colaborativos con TIC. Análisis de dos experiencias en el contexto educativo. EDUTEC, Revista Electrónica de Tecnología Educativa, 44. DOI: 10.21556/edutec.2013.44.324.

- Belloch, C. (S.F.). Los contenidos en los EVA. Valencia: Universidad de Valencia. Recuperado de: http://www.uv.es/bellochc/pedagogia/EVA6.pdf.

- Boneu, J. N. (2007). Plataformas abiertas de elearning para el soporte de contenidos educativos abiertos. Revista de Universidad y Sociedad del Conocimiento. 4(1), 36-46. Recuperado de: https://dialnet.unirioja.es/descarga/articulo/2291 412.pdf.

- Cabero, J. (2010). Los retos de la integración de las TICs en los procesos educativos. Límites y posibilidades. Perspectiva educacional. 49 (1), 3261. Recuperado de: http://www.perspectivaeducacional.cl/index.php/ peducacional/article/view/3.

- Cabero, J. (2015). Tendencias para el aprendizaje digital: de los contenidos cerrados al diseño de materiales centrado en las actividades. El proyecto Dipro 2.0. Revista de Educación a Distancia, 32,127. Recuperado de: http://revistas.um.es/red/article/view/233041.

- Cabezas, M., Casillas, S. y Pinto, A. M. (2014). Percepción de los alumnos de Educación Primaria de la Universidad de Salamanca sobre su competencia digital. EDUTEC, Revista Electrónica de Tecnología Educativa. 48, 1-14. Recuperado de: http://www.edutec.es/revista/index.php/edutece/article/view/156/21.
- Calderone, M. y González, A.H. (2016). Materiales didácticos. Una metodología para su producción en la era de las TIC. Virtualidad, Educación y Ciencia. 7 (13), 24-35. Recuperado de: https://revistas.unc.edu.ar/index.php/vesc/article /viewFile/16204/16081.

- Castañeda, L., Esteve, F. y Adell, J. (2018). ¿Por qué es necesario repensar la competencia docente para el mundo digital? En Revista de Educación a Distancia, 56, 1-20. DOI: 10.6018/red/56/6.

- Castaño, C. (2013). Tecnologías y medios para la educación en la e-sociedad. Los recursos de la Web 2.0: su utilización educativa. En Aguaded, J. I. y Cabero, J. (Coords.).Tecnologías y medios para la educación en la e-sociedad, pp. 53-70. Madrid: Alianza Editorial.

- Cebrián, M. (2013). Juegos digitales para procesos educativos. En Aguaded, J.L. y Cabero, J. (coords). Tecnologías y medios para la educación en la ESociedad, pp. 185-210. Madrid: Alianza Editorial.

- Colás, M., de Pablos, J., y Ballesta, J. (2018). Incidencia de las TIC en la enseñanza en el sistema educativo español: una revisión de la investigación. Revista de Educación a Distancia, 56, 1-56. Recuperado de: https://revistas.um.es/red/article/view/321471.

- Coll, C. (2004). Psicología de la educación y prácticas educativas mediadas por las tecnologías de la información y la comunicación: una mirada constructivista. Sinéctica, 25, 1-24. Recuperado de: http://www.redalyc.org/articulo.oa?id=99815899 016.

- Cortés, M. (2013). La integración de las TAC en Educación (Trabajo de fin de grado). Universidad Internacional de La Rioja, Valencia. Recuperado de: http://reunir.unir.net/handle/123456789/1846.

- Dorfsman, M. (2018). El educador en línea: más allá de la digitalidad. Revista de Educación a Distancia, 56,1-25. DOI: 10.6018/red/56/9.

- Dorrego, E. (2016). Educación a distancia y evaluación del aprendizaje. Revista de Educación a Distancia, 50, 1-18.

- Enríquez, S. (2012). La formación básica para docentes y las TAC. Educación y TIC. Recuperado de: http://www.ead.unlp.edu.ar/blog/?p=209.

- Expósito, J. y Manzano, B. (2013). Escuela TIC 2.0: aprendizaje del alumnado de primaria en contextos educativos y socio familiares. EDUTEC, Revista Electrónica de Tecnología Educativa, 45.

Recuperado de: http://www.edutec.es/revista/index.php/edutece/article/view/30.

- Farías, M. y Araya, C. (2014). Alfabetización visual crítica y educación en lengua materna: estrategias metacognitivas en la comprensión lectora de textos multimodales. Colombian Applied Linguistics 
Journal, 16(1), 93-104. DOI:

10.14483/udistrital.jour.calj.2014.1.a08.

- Fernández, M. C. y Cebreiro, B. (2003). Evaluación de la enseñanza con TIC. Pixel-Bit. Revista de Medios y Educación, 21, 65-72.

- García, F. y Ruiz de Adana, M. A. (2013). Las TIC en la escuela. Teoría y práctica. Barcelona: Editorial ECU.

- García-Valcárcel, A. y Tejedor F.J. (2010). Evaluación de procesos de innovación escolar basados en el uso de las TIC desarrollados en la Comunidad de Castilla y León. Revista de Educación, 352, 125-147. Recuperado de: http://www.revistaeducacion.educacion.es/re352 /re352_06.pdf.

- Gewerc, A. (2002). ¿Qué hacen estos bits en mi aula? Posibilidades educativas de los materiales multimedia. En Vez, J.M. (Dir.), Aplicaciones de las nuevas tecnologías en el aprendizaje de la lengua castellana, 117-141. Madrid: Ministerio de Educación, Cultura y Deporte.

- Kress, G., Jewitt, C., Ogborn, J. y Tsatsarelis, C. (2001). Multimodal Teaching and Learning: Rhetorics of the Science Classroom. London: Continuum.

- Martí, J. y Muñoz, P. (2008). Engagement marketing: una nueva publicidad para un marketing de compromiso. Madrid: Financial Times/Prentice Hall.

- Martín, C (2013). Didáctica de la educación infantil. Madrid: Macmillan Iberia.

- Martín, E. y Marchesi, A. (2006). La integración de las Tecnologías de la Información y la Comunicación en los Sistemas Educativos: Propuestas de introducción en el currículum de las competencias relacionadas con las TIC. Buenos Aires: Sede Unesco de Buenos Aires. Recuperado de:

http://unesdoc.unesco.org/images/0015/001507/ 150785s.pdf.

- Mauri, T., Onrubia, J., Coll, C., y Colomina, R. (2016). La calidad de los contenidos educativos reutilizables: diseño, usabilidad y prácticas de uso. Revista De Educación a Distancia. Recuperado de: https://revistas.um.es/red/article/view/25091.

- Minelli de Oliveira, J. (s.f.). Resultados de la práctica docente según la percepción de los estudiantes: La formación de maestros en la era digital. Recuperado de:

http://www.quadernsdigitals.net/datos/hemerote ca/r_1/nr_804/a_10847/10847.html .

- Onrubia, J. (2016). Aprender y enseñar en entornos virtuales: actividad conjunta, ayuda pedagógica y construcción del conocimiento. Revista de Educación a Distancia, 50,1-14. DOI: $10.6018 / \mathrm{red} / 50 / 3$.
- Real Decreto n 126. Currículo básico de la Educación Primaria, Boletín Oficial del Estado, Madrid, núm. 52, 1 de marzo de 2014.

- Real Decreto no 1630. De enseñanzas mínimas del segundo ciclo de Educación infantil. Boletín Oficial del Estado. Madrid, núm. 4, 4 de enero de 2007, pp. 474-482.

- Rey, R., Barbosa, J.W., Gómez, G. (2003). Evaluación de materiales escritos de autoaprendizaje para educación a distancia. Revista Iberoamericana de Educación a Distancia. 6(2), 55-75. DOI: $10.5944 /$ ried.6.2.1096.

- Rocha, G. y Ferreira, C.A. (2012). Desenho de conteúdos e-learning: quais teorias de aprendizagem podemos encontrar? Revista Internacional de Educación a Distancia, RIED. 15 (1). DOI: 10.5944/ried.1.15.778.

- Sáinz de Vicuña, P. (2009). Una propuesta metodológica para el aula de niños y niñas de 2 años. CEE Participación Educativa, 12, 35-55.

- Salmerón, H., Rodríguez, S. y Gutiérrez, C. (2010). Metodologías que optimizan la comunicación en entornos de aprendizaje virtual. Comunicar, Revista Científica de Educomunicación, 34 (XVII), 163-171. DOI: 10.3916/C34-2010-03-16.

- Silva, J. (2017). Un modelo pedagógico virtual centrado en la E-actividades. En Revista de Educación a Distancia, 53, 1-20. DOI: 10.6018/red/53/10.

- Siraj-Blatchford, J. (Comp.) (2005). Nuevas tecnologías para la Educación Infantil y Primaria. Madrid: Ministerio de Educación y CienciaEdiciones Morata.

- UNESCO (2008). Estándares de competencia en TIC para docentes. París: Organización de las Naciones Unidas para la Educación, la Ciencia y la Cultura.

- Urbina. S. (2006). Algunas consideraciones en torno al software para Educación Infantil. Edutec. Revista Electrónica de Tecnología Educativa, 13. DOI: 10.21556/edutec.2000.13.554.

- Zapata, M. (2016). Secuenciación de contenidos y objetos de aprendizaje. En Revista de Educación a Distancia, 50, 1-29. Recuperado de: http://revistas.um.es/red/article/view/25221/24 521. 